\title{
EFFECTS OF LOW TEMPERATURE ON THE SOMATIC INSTABILITY OF AN ALIEN CHROMOSOME IN NICOTIANA TABACUM
}

\author{
Z. LUCOV, S. COHEN and R. MOAV \\ Department of Genetics, The Hebrew University of Jerusalem, Jerusalem, Israel
}

Received 20.ix.69

\section{INTRODUGtion}

IT has been shown that interspecific hybridisation frequently interferes with the orderly behaviour of chromosomes in somatic tissues (Rees, 1961; Gerstel and Burns, 1966; Gopinath et al., 1965). Moav (1961) and Shargal and Moav (1967) have reported that colour variegation in Nicotiana tabacum and in some hybrid derivatives of Nicotiana is a consequence of somatic chromosome elimination. Sand (1956 and 1957) have shown that low temperatures increase the rate of colour variegation in some Nicotiana hybrid derivatives.

In the present investigation, the somatic instability of an alien univalent of $\mathcal{N}$. paniculata in a $\mathcal{N}$. tabacum nucleus was compared to that of a normal chromosome in $\mathcal{N}$. tabacum, when grown under optimal temperature in a greenhouse, and when exposed to lower temperatures.

\section{Plant material}

The two species involved in this study were Nicotiana tabacum variety Red Russian, $n=24$ (henceforth $t b c$ ), and $\mathcal{N}$. paniculata, $n=10$ (henceforth $p n c)$. The two species are described by Goodspeed (1954).

Heterozygous alien addition plants possessing all the 24 bivalents of $t b c$ plus a marked univalent of pnc were obtained from the Department of Genetics, University of California, Berkeley. Their tbc chromosomal complement was homozygous for the recesive mutant ws (white seedling) which in the homozygous condition inhibits chlorophyll formation. The alien univalent carried the dominant normal allele $W s$ (White seedlingNormal) and was designated $W s(p n c)$.

Chromosomally normal tbc plants heterozygous for the $W s$ locus (henceforth designated $W s(t b c)$ were used for controls.

\section{EXPERIMENTAL PROCEDURE}

All the seeds were germinated in the laboratory on wet filter paper in petri dishes. After germination, they were transferred to pots and placed in a greenhouse. Cold treatment was given when the plants were about eight weeks old and after they had developed several true leaves.

The presence of at least a single dominant $W s$ allele is necessary for chlorophyll production. Therefore, when the $W_{s}$ (pnc) univalent of the heterozygous alien addition plants or the $W_{s}(t b c)$ chromosome of the heterozygous $W s$, ws tbc plants were lost in somatic divisions, or when the marker had undergone a point mutation to the recessive condition, the 


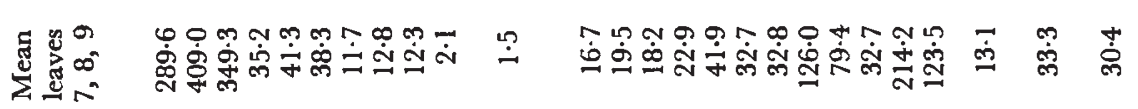

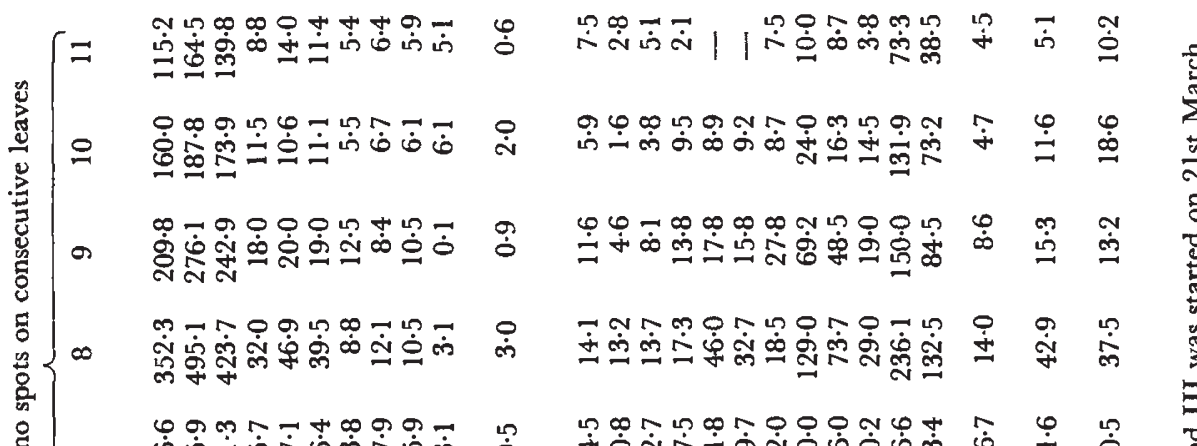

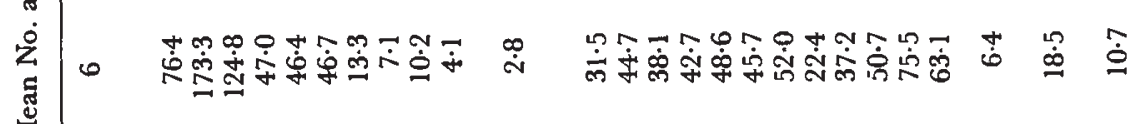

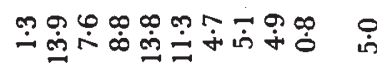

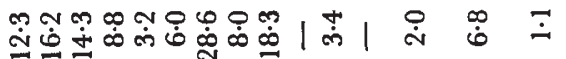

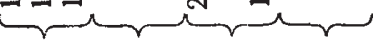

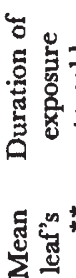

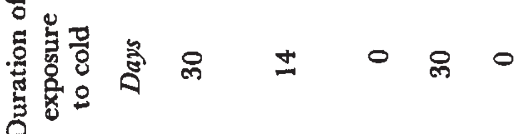
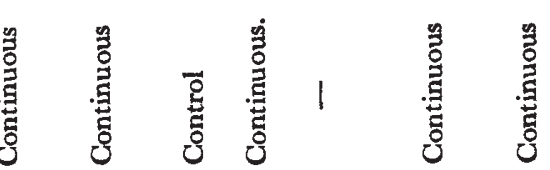

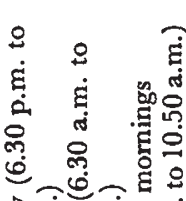

m 0

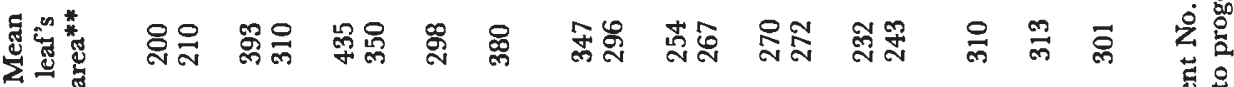

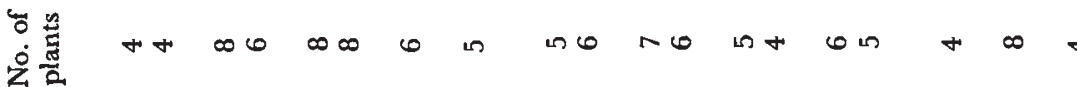

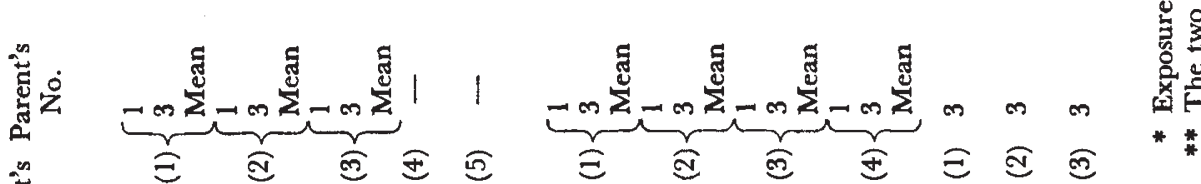


resulting cell lineage was incapable of producing chlorophyll. If the loss took place in a green tissue, an albino (chlorophylless) spot developed from the resulting cell lineage. The number of albino spots per unit leaf area served as a measure of the degree of instability of the marked chromosomes.

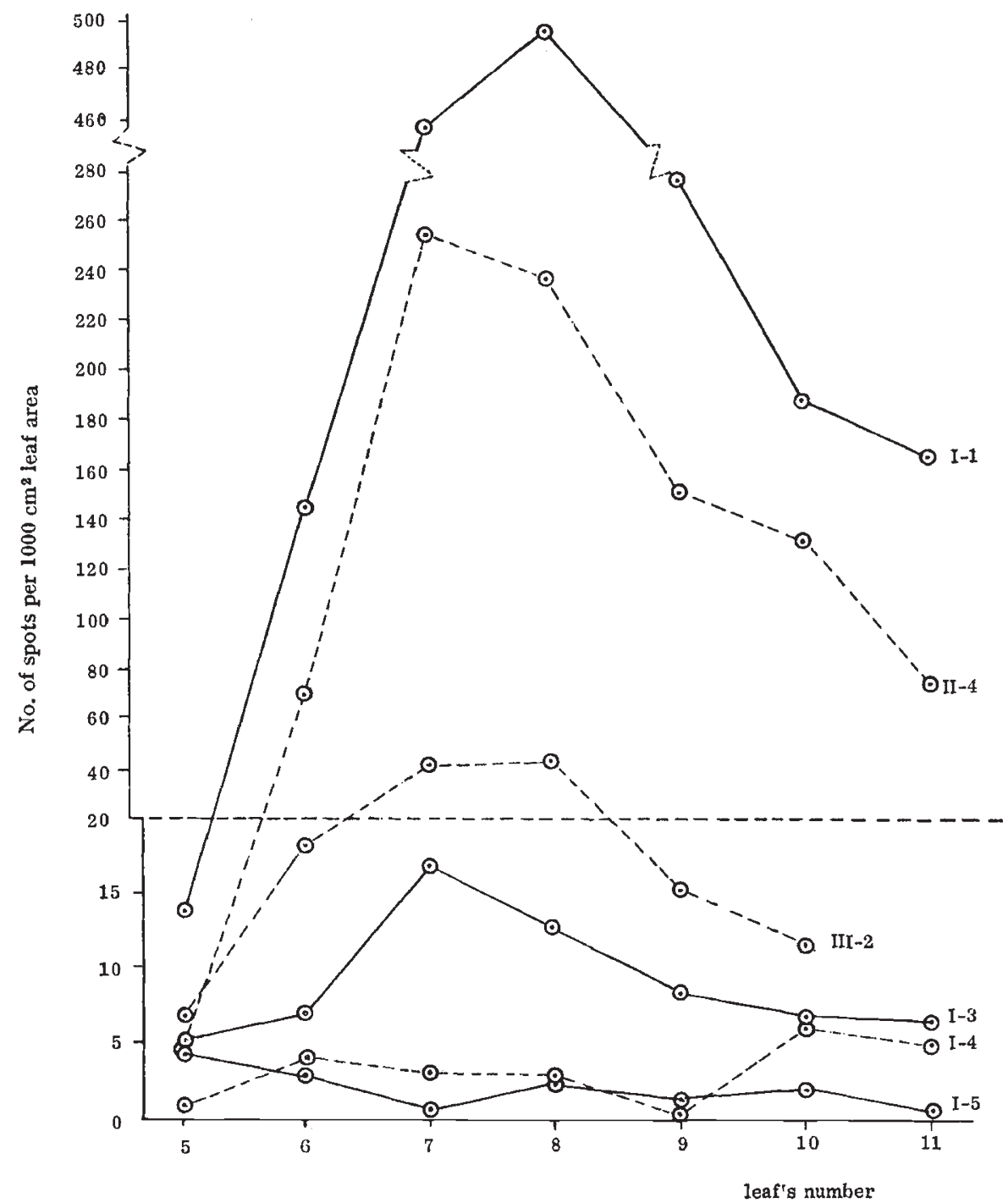

Fig. 1.-Frequencies of albino leaf spots in the first three experiments. (For details and explanations of the curves designations see table 1.)

Four experiments were carried out, the first three in 1959 (table 1 and fig. 1) and the fourth in 1962 (figs. 2 and 3). The plants used in the 1962 experiment were the direct progeny of plants tested in 1959.

In 1959, the cold treatment consisted of taking the plants out of the greenhouse for various periods. The minimum temperature in the greenhouse during that period was $15^{\circ} \mathrm{C}$., while the outside average minimum and maximum daily temperatures were $6 \cdot 9^{\circ} \mathrm{C}$. and $14 \cdot 4^{\circ} \mathrm{C}$. respectively. 
In 1962, the exposure to cold was carried out in a dark refrigerator with a temperature range of $2^{\circ} \mathrm{C}$. to $6^{\circ} \mathrm{C}$. The duration of the cold treatments was 14 days. Subsequently, plants were transplanted into a fertile field where they developed into large plants with many large leaves.

The measure of instability. The degree of instability of the chromosomes marked by $W s$ was measured by counting the number of albino (chlorophylless) spots with the naked eye. The counting was done when the leaves reached their full size.

Measurements of length and width of 100 leaves in a random sample showed that the ratio width/length was 0.59 with a rather small variance. Therefore, only the length of the tested leaves was measured. In the first three experiments, the unit of comparison was: number of spots per $1000 \mathrm{~cm} .^{2}$ of calculated leaf area, when the "calculated area" was defined as 0.59 multiplied by the leaf's length. In the fourth experiment, the unit of comparison was: number of spots per $10 \mathrm{~cm}$. leaf length.

Progeny testing. Progeny testing was used in order to ascertain that the plants used in the experiments had the proper genotypes. Each plant was selfed and the segregation at the $W s$ locus (green $v$ albino seedlings) was determined in a random sample of approximately 100 seedlings.

Cytological examination of pollen mother cells of some heterozygous alien addition plants was used to verify that the unmodified alien univalent was present.

\section{EXPERIMENTAL RESULTS}

Preliminary experiment. Ten $W_{S}(p n c)$ heterozygous alien addition plants (containing all the $24 t b c$ bivalents homozygous recessive $w s$, ws plus a pnc univalent carrying the dominant $W s$ allele) were grown in pots outdoors during the late winter. The leaves of all the 10 plants were mottled with many albino spots (100 to 200 spots per leaf). The stems of all these plants were cut to about $6 \mathrm{~cm}$. above pot level. A random sample of five plants was placed in the greenhouse, while the remaining five were left outdoors and each plant was allowed to develop a single side-shoot. The side-shoots of all the plants grown outdoors were again mottled with many spots, but the side-shoots of the plants kept in the greenhouse showed very few spots.

This preliminary experiment showed that in somatic tissues, the alien $W s$ (pnc) chromosome was sensitive to cold and that the new tissues developed after the return of the plants to higher temperature did not retain this sensitivity.

The rate of spotting due to loss of the normal $\mathrm{Ws}$ (tbc) chromosome. Two samples of chromosomally normal tbc plants, heterozygous for the $W s$ marker $(W s, w s)$ were grown. One sample was exposed to outside temperatures for 30 days and the second sample remained in the greenhouse (table 1, treatments I (4) and I (5) respectively). The rates of spotting of these two samples are presented in fig. 1 (the two lower curves-I (4) and I (5)). It appears that outdoor conditions did not have any detectable effect on the rate of spotting of the normal $t b c$ chromosomes.

The rate of spotting due to loss of the alein Ws (pnc) chromosome of two different parents. Each treatment, in the first two experiments (table 1, I and II), was given to four replicated samples of the heterozygous alien addition plants $W s(p n c)$. Two of the four samples originated from two capsules of one parent (Parent No. 1) and the remaining two from two capsules of a second parent 
(Parent No. 3). While the results obtained from two samples of the same parent were highly uniform, and therefore pooled, a wide difference existed between the two parents. The offspring of Plant No. 3 consistently showed a considerably higher rate of spotting than those of Plant No. 1 (table 1). A probable cause of this difference is a spontaneous change in the alien $W s$ (pnc) chromosome of the parent (Moav, 1961).

The rate of spotting due to loss of the alien Ws (pnc) in the greenhouse. The rate of spotting of the alien $W s(p n c)$ when grown in the greenhouse was approximately four times higher than that of the normal $W_{s}(t b c)$ (table 1, experiment I, or compare curves I (3) with I (4) and I (5) in fig. 1). While the rate of spotting of the latter remained more or less constant, the rate of spotting of $W s(p n c)$ showed an increase of spots up to leaf No. 7 and then a decline till leaf No. 10. It should be pointed out that the greenhouse was not heated properly, so that night temperatures were lower than that considered optimal for tobacco plants. Thus, it may be speculated that under optimal temperature conditions, the level of spotting of the alien $W_{s}$ (pnc) would have been more similar to that of the normal $W_{s}(t b c)$.

The rate of spotting of the alien Ws (pnc) under continuous exposure to outdoor conditions. Experiment I shows the response of $W_{s}(p n c)$ to continuous exposure to outdoor conditions for 14 and 30 days (see curve I (1) in fig. 1), while experiment II shows the response to exposure for 3, 6, 15 and 20 days. The two experiments show that leaf No. 5 was not affected by the treatment, leaf No. 6 was partially affected, and that the effect increased up to leaf No. 8, after which it began to decline, reaching the lowest level in leaf No. 11.

Our interpretation of these results is that in leaf No. 5 most of the cell divisions had already taken place when exposure to outdoor conditions was started. The proportion of cell divisions that took place during exposure increased in subsequent leaves until it reached a peak in leaf No. 8, after which this proportion had gradually declined until it once again reached zero.

The rate of spotting of the alien Ws (pnc) under discontinuous exposure to outdoor conditions. For experiment III, only the offsprings of one parent (plant, No. 3) were used. The three treatments in this experiment (table 1 and curve III (2) in fig. 1) consisted of exposing the plants to outdoor conditions at different times of the day. For the remaining time they were kept in the greenhouse. The results of this experiment may be summed up as follows:

(a) Contrary to expectations, night exposure alone did not increase the rate of spotting (compare treatments III (1) and I (3)).

(b) Exposure at night and in the morning hours and exposure during the daytine only, showed a considerable, and almost identical, response (III (2) and III (3)). This probably means that exposure during the morning hours is most effective, despite the fact that the morning temperature is higher than night temperatures.

(c) Continuous exposure to outdoor conditions for the same duration of time (II (3)) yielded many more spots (about three times as many as at the peak) than the most effective discontinuous exposure.

A likely interpretation of these results is that there are some sensitive stages of cell division which are more frequent during the morning hours. This would mean that in all cases of discontinuous exposure, a high proportion of the sensitive stages of cell division occurred while the plants were in the greenhouse and they were therefore not affected by the exposure. 
The rate of spotting of the alien Ws (pnc) under discontinuous exposure to cold in a refrigerator. Fig. 2 shows the various cold-plus-darkness treatments in experiment IV, and fig. 3 shows the effects of these treatments on the rate of spotting. The unit of measurement was the number of spots per $10 \mathrm{~cm}$. of leaf length. The results of this experiment cannot therefore be compared directly with those of the earlier experiments.

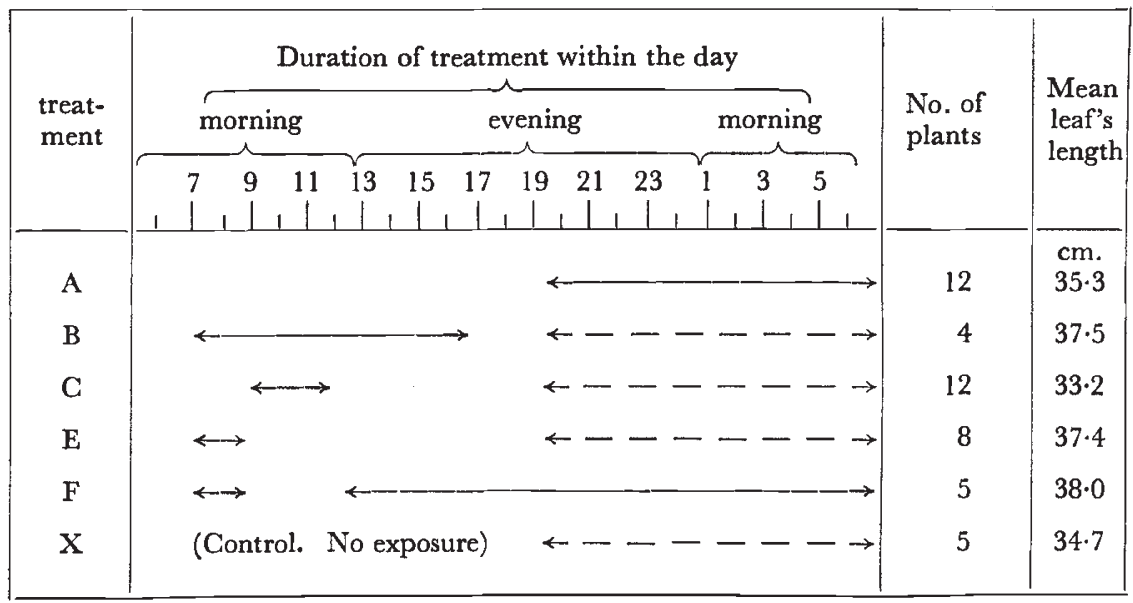

FIG. 2.-The various cold treatments of the 1962 experiments. (All the tested plants were alien addition possessing the $W s(p n c)$ chromosome. Solid lines indicate exposure to cold and broken lines, darkness.)

"Running means" (fig. 3) were used in order to avoid large, random, fluctuations from leaf to leaf. The results of this experiment may be summed up as follows:

(a) All the cold treatments responded with an increased number of spots.

(b) Exposure during the day (treatment B) was more effective than exposure during the night (treatment $\mathrm{A}$ ) or than exposure during the night plus afternoon (treatment $F$ ).

(c) Exposure for only three hours before noon (from 9 to 12 a.m., treatment $\mathrm{C}$ ) was almost as effective as exposure for 21 hours extending over the whole afternoon and night (from 12 noon till 9 a.m. the next day-treatment $\mathrm{F}$ ).

(d) Exposure for only two hours in the early morning following 12 hours of darkness (treatment E) had almost no effect.

The degree of sensitivity of this experiment was not sufficient to resolve fully the relationship between the rate of spotting and the time and duration of exposure to low temperature and light. Nevertheless, the experiment clearly demonstrated that the interaction between all three factors is important and that exposure before noon is most effective. It also showed that "shock" treatments of only 2 hours (treatment E) have hardly any effect on the frequency of spotting. A probable interpretation is that a small proportion of cells were in their sensitive stage during the cold "shock" and that the treatment had no after-effects.

The rate of spotting when two Ws markers were present in the same nucleus. Not 
a single "countable" spot was found whenever two Ws markers were present within the same plant, regardless of whether the two were of $t b c$, or of pnc or of one of each, and also regardless of whether the plants were grown in the greenhouse or outside. This result is to be expected if it is accepted that the $W s$ allele must be missing from albino tissue and that the elimination

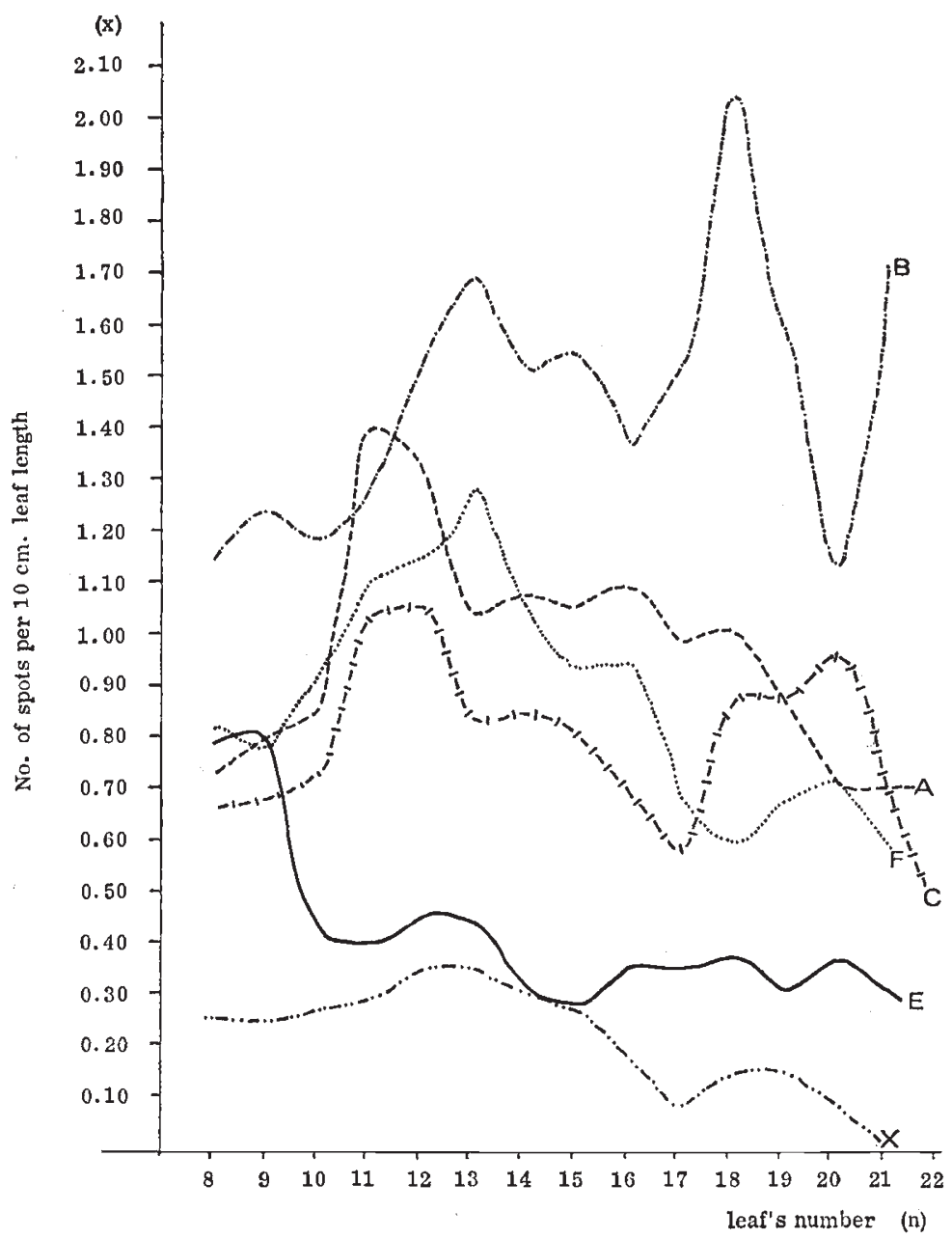

FIG. 3.-Rates of spotting of consecutive leaves at the various cold treatments of the 1962 experiment. (Running means: $(1 / 3)\left(X_{n-1}+X_{n}+X_{n+1}\right)$ were used to smooth the curves. Description of the symbols $\mathrm{A}, \mathrm{B}, \mathrm{C}, \mathrm{E}, \mathrm{F}$ and $\mathrm{X}$ is given in fig. 2.)

rate of the $W s$ is very low even in the most effective cold treatment, so that the rate of elimination of two $W s$ alleles from the same cell lineage is almost zero.

\section{Discussion}

Earlier work strongly indicated that the present albino spots are a consequence of somatic elimination of chromosomes rather than of somatic mutations (Moav, 1961; Gupta, 1968; Shargal and Moav, 1967). Accepting 
this hypothesis means that each spot represents the cell lineage of a single and usually independent loss of a chromosome. The independence of the events leading to the albino spots is concluded from the more or less random distribution of the spots.

Three conclusions can be made regarding the effects of low temperature on the somatic instability of the alien $W s(p n c)$ chromosome. These are:

(a) While exposure to cold increases many times the degree of somatic instability of the alien chromosome, it apparently has no effect on the somatic stability of the host's chromosomes. This points to a mechanism that preserves the regularity of replication of the whole chromosomal set when under stress. When chromosomes of one species are introduced by introgression into a nucleus of another species, their regulation by the host's mechanism is occasionally not fully effective and they are therefore lost at higher frequencies when exposed to an adverse environment.

(b) Low temperature exerts its effects only during the period of exposure and has no after-effects when the plants are returned to optimal temperature conditions, i.e. the greenhouse.

(c) The chromosomes are affected only at some specific sensitive stage of their replication division cycle (Collins, 1968). It should be noted that the alien addition plants (24 II $(t b c)+$ I $W s(p b g)$ ) differed from their control (chromosomally normal $t b c$, heterozygous at the $W s$ locus), not only in that they had the $W_{s}$ marker, or an alien chromosome, but also in that they were in a trisomic condition. Therefore, the present evidence cannot rule out trisomy per se as a possible cause of increased chromosome sensitivity to exposure.

\section{Summary}

1. Nicotiana tabacum plants heterozygous for the recessive gene ws (white seedling which in homozygous condition prevents chlorophyll production) show occasional albino (chlorophylless) spots on their leaves. Each spot probably originates from a somatic loss of the chromosome carrying the dominant allele $W s$.

2. Exposure of heterozygous plants to lower temperatures did not alter the number of albino spots. When, however, the dominant $W s$ allele was carried by an alien chromosome, introduced from $\mathcal{N}$. paniculata, and the two $\mathcal{N}$. tabacum genomes possessed the recessive ws, the number of albino spots increased manifold.

3. This points to a mechanism that preserves the stability of the host's chromosomes when they are exposed to adverse conditions, but fails to do so efficiently to the alien chromosome.

4. By exposing the alien addition plants to cold during different times of the day, it was found that complex interactions between temperature, light and the time of the day at which the exposure takes place account for a large variation in the number of spots.

5. It was also concluded that the exposure is only effective at certain "sensitive" stages of cell division and that it has no after-effects.

Acknowledgment.-The writers wish to express their gratitude to Professor D. R. Cameron for his generosity in supplying the seeds needed for the present investigation. 


\section{REFERENGES}

Collins, G. B. 1968. DNA synthesis in two speies of Nicotiana and their hybrid. F. Herd., $59(1), 7-13$.

GERSTEL, D. U., AND BURNS, J. A. 1966. Flower variegation in hybrids between $\mathcal{N}$. tabacum, and $\mathcal{N}$. otophora. Genetics, 53(3), 551-567.

Goodspeed, T. H. 1954. The genus Nicotiana. Chronica Botanica Comp.

GOPINATH, D. M., KRISHNAMURTHY, K. R., AND SUBHASHINI, L. 1965. On the chromosome loss in interspecific hybrids of Nicotania. Nucleus, 8(2), 171-178.

GUPTA, S. B. 1968. Chlorophyll variegation caused by somatic elimination of an alien chromosomal fragment in Nicotiana tabacum. Canadian F. of Genetics and Cytology, 10(1), 106-111.

GUPTA, s. B. 1968. Chlorophyll variegation caused by unstable behaviour of an alien chromosome in hybrid derivatives of Nicotiana species. Genetics, 59, 193-208.

MOAv, R. 1961. Genetic instability in Nicotiana hybrids. II. Studies of the $W s(p b g)$ locus of $\mathcal{N}$. plumbaginifolia in $\mathcal{N}$. tabacum nuclei. Genetics, 46(9), 1069-1088.

REES, H. 1961. Genotypic control of chromosome form and behaviour. Bot. Rev., 27, 288318.

SAND, s. A. 1956. Correlated effects of temperature on somatic and germinal mutation in a clone of variegated Nicotiana. Genetics, $41(5), 659$.

SAND, s. A. 1957. Phenotypic variability and the influence of temperature on somatic instability in cultures derived from hybrids between $\mathcal{N}$. langsdorffi and $\mathcal{N}$. sanderae. Genetics, 42(6), 685-703.

SHARGAL, M., AND MOAV, R. 1967. Cytological analysis of mutant albino seedlings in Nicotiana tabacum. Heredity, 22(2), 304-306. 